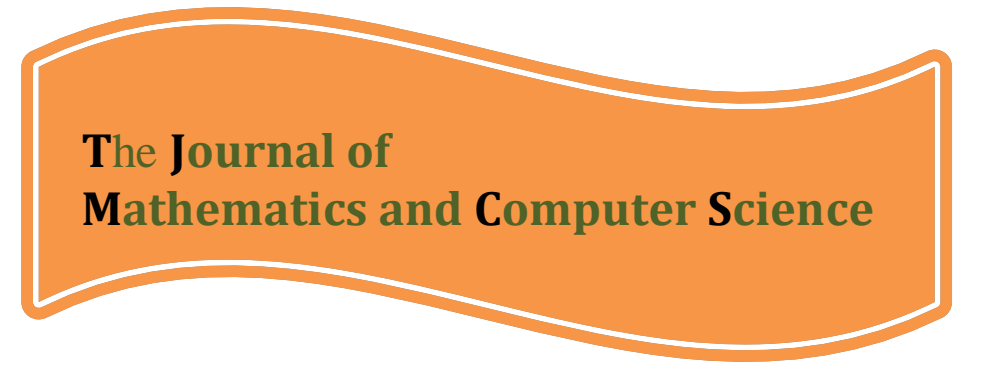

Available online at

http://www.TJMCS.com

The Journal of Mathematics and Computer Science Vol .2 No.4 (2011) 631-638

\title{
Some Remarks on Manifolds with Vanishing Bochner Tensor
}

\author{
Eileen May G. Cariaga1, Richard S. Lemence ${ }^{2, \#}$ and Marian P. Roque ${ }^{3}$
}

Institute of Mathematics, College of Science, University of the Philippines, Diliman, Quezon City, Philippines 1ayen.cariaga@gmail.com,

2rslemence@gmail.com,

${ }^{3}$ marian.roque11@gmail.com

Received: August 2010, Revised: November 2010

Online Publication: January 2011

\begin{abstract}
In this paper, we present our results on Einsteinian, almost Hermitian manifolds with Bochner tensor $B=0$. It shall be shown that under some conditions, these Bochner flat manifolds are complex space forms. Moreover, they are also Kähler manifolds with a constant holomorphic sectional curvature. We also present an identity for the Riemannian curvature of a generalized complex space form.
\end{abstract}

Keywords: Complex space forms, Kähler manifolds, Bochner tensor, generalized complex space forms

AMS Subject Classification (MSC2010): 53C25, 53C56

\section{Preliminaries}

Let $M=(M, J, g)$ be a $2 n$-dimensional almost Hermitian manifold with the almost complex structure $J$ and Riemannian metric $g$. Let $\nabla$ be the Levi-Civita connection on $M$ and $R$ the Riemannian curvature tensor defined by

\footnotetext{
\# Currently, he is a postdoctoral research fellow at the Academic Production, Ochanomizu University, Bunkyo-ku, Tokyo, Japan.
} 
Eileen May G. Cariaga, Richard S. Lemence, Marian P. Roque/ TJMCS Vol .2 No.4 (2011) 631-638

$$
R(X, Y) Z=\nabla_{X}\left(\nabla_{Y} Z\right)-\nabla_{Y}\left(\nabla_{X} Z\right)-\nabla_{[X, Y]} Z
$$

for $X, Y$ and $Z \in \Gamma(M)$, where $\Gamma(M)$ denotes the Lie algebra of all smooth vector fields of $M$.

The Ricci tensor $\rho$ on $M$ is a symmetric bilinear function on $\Gamma(M) \times \Gamma(M)$ defined as the trace of the mapping $T: \Gamma(M) \rightarrow \Gamma(M)$ given by $T(\mathrm{Z})=R(X, Z) Y$. Equivalently,

$$
\begin{aligned}
\rho(X, Y) & =\operatorname{trace}(T) \\
& =\sum_{i=1}^{n} R\left(X, e_{i}, Y, e_{i}\right)
\end{aligned}
$$

where $\left\{\mathrm{e}_{\mathrm{i}} \mid \mathrm{i}=1, \ldots, \mathrm{n}\right\}$ is an orthonormal basis of $T_{p}(M)$, the tangent space of $M$ at the point $p$. The Ricci tensor transformation $Q=Q(R)$ is given by $\rho(X, Y)=g(Q X, Y)$ and the trace of $Q$ is called the scalar curvature $\tau=\tau(R)$ of $R$. Note that since $\rho$ is symmetric, then for vectors $X, Y \in \Gamma(M)$,

$$
\rho(X, Y)=\rho(Y, X) .
$$

Furthermore, we denote by $\rho^{*}$ and $\tau^{*}$ the Ricci ${ }^{*}$-tensor and the ${ }^{*}$-scalar curvature on $M$, respectively. The tensor $\rho^{*}$ is defined pointwisely by

$$
\begin{aligned}
\rho^{*}(X, Y) & =\operatorname{trace}(Z \text { a } R(J Z, X) J Y) \\
& =-\sum_{i=1}^{2 n} R\left(X, e_{i}, J Y, J e_{i}\right) \\
& =-\frac{1}{2} \sum_{i=1}^{2 n} R\left(X, J Y, e_{i}, J e_{i}\right)
\end{aligned}
$$

where $X, Y$ and $Z \in T_{p}(M), R(X, Y, Z, W)=g(R(X, Y) Z, W)$ and $\left\{e_{i}\right\}$ is an orthonormal basis of $T_{p}(M)$. We also define analogously the Ricci ${ }^{*}$-operator, denoted by $Q^{*}$, by $\rho^{*}(X, Y)=g\left(Q^{*} X, Y\right)$ for $X$ and $Y \in T_{p}(M)$. The trace of $Q^{*}$ is the ${ }^{*}$-scalar curvature $\tau^{*}$ on $M$. We note that $\rho^{*}$ satisfies $\rho^{*}(J X, J Y)=\rho^{*}(Y, X)$ but is not symmetric in general.

A manifold $M$ is called an Einstein space or Einsteinian if $\rho=\lambda g$ for some constant $\lambda$. It is easy to check that the Einstein constant $\lambda$ is equal to $\frac{\tau}{m}$, where $m$ is the dimension of the manifold and so $\rho=\frac{\tau}{m} g$. A space of constant curvature is necessarily an Einstein space. In fact, for $m$-dimensional space of constant curvature $\lambda, g=\lambda(m-1) \rho$. An almost Hermitian manifold $M$ is called a weakly *Einstein manifold if $\rho^{*}=\frac{\tau^{*}}{2 n} g(\operatorname{dim} M=2 n)$ holds. In addition, if $\tau^{*}$ is constantvalued, then $M$ is called *-Einstein manifold. 
Eileen May G. Cariaga, Richard S. Lemence, Marian P. Roque/ TJMCS Vol .2 No.4 (2011) 631-638 given by

We now define the Bochner tensor $B$. Let $R_{J}$ denote the curvature tensor

$$
R_{J}(X, Y, Z, W)=\mathrm{R}(J X, J Y, J Z, J W)
$$

$\pi_{1}$ and $\pi_{2}$ the tensors defined by

$$
\begin{gathered}
\pi_{1}(X, Y, Z, W)=g(X, Z) g(Y, W)-g(Y, Z) g(X, W) \\
\pi_{2}(X, Y, Z, W)=2 g(J X, Y) g(U Z, W)+g(J X, Z) g(Y Y, W)-g(U Y, Z) g(J X, W)
\end{gathered}
$$

and for $S \in(\Gamma(M) \times \Gamma(M))^{*}$, we define the functions $\varphi(S)$ and $\psi(S)$ by

$$
\begin{aligned}
\varphi(S)(X, Y, Z, W)= & g(X, Z) S(Y, W)+g(Y, W) S(X, Z) \\
& -g(X, W) S(Y, Z)-g(Y, Z) S(X, W) \\
\psi(S)(X, Y, Z, W)= & 2 g(X, J Y) S(Z, J W)+2 g(Z, J W) S(X, J Y) \\
+ & g(X, J Z) S(Y, J W)+g(Y, J W) S(X, J Z) \\
& -g(X, J W) S(Y, J Z)-g(Y, J Z) S(X, J W)
\end{aligned}
$$

where $\Gamma^{*}(M)$ denotes the dual space of $\Gamma(M)$. The Bochner tensor associated with the curvature tensor $R$ is given by

$$
\begin{aligned}
B(R)(X, Y, Z, W)= & R(X, Y, Z, W)-\frac{1}{16(n+2)}(\varphi+\psi)\left(\rho+3 \rho^{*}\right)\left(R+R_{J}\right)(X, Y, Z, W) \\
& -\frac{1}{16(n-2)}(3 \varphi-\psi)\left(\rho-\rho^{*}\right)\left(R+R_{J}\right)(X, Y, Z, W) \\
& -\left\{\frac{1}{4(n+1)}\left(\psi \circ \rho^{*}\right)+\frac{1}{4(n-1)}(\varphi \circ \rho)\right\}\left(R-R_{J}\right)(X, Y, Z, W) \\
& +\frac{1}{16(n+1)(n+2)}\left(\tau+3 \tau^{*}\right)(R)\left(\pi_{1}+\pi_{2}\right)(X, Y, Z, W) \\
& +\frac{1}{16(n-1)(n-2)}\left(\tau-\tau^{*}\right)(R)\left(3 \pi_{1}-\pi_{2}\right)(X, Y, Z, W)
\end{aligned}
$$

for $n \geq 3$, and by

$$
\begin{aligned}
B(R)(X, Y, Z, W)= & R(X, Y, Z, W)-\frac{1}{2} \varphi\left(\rho(R)-\frac{1}{4} \tau(R) g\right)(X, Y, Z, W) \\
& -\frac{1}{12} \psi\left(\rho^{*}\left(R-R_{J}\right)\right)(X, Y, Z, W) \\
& -\frac{1}{96}\left(\tau+3 \tau^{*}\right)(R)\left(\pi_{1}+\pi_{2}\right)(X, Y, Z, W) \\
& -\frac{1}{32}\left(\tau-\tau^{*}\right)(R)\left(3 \pi_{1}-\pi_{2}\right)(X, Y, Z, W)
\end{aligned}
$$

for $n=2$. 
If the manifold has a vanishing Bochner tensor, i.e., $B=0$, then it is called a Bochner-flat manifold.

The concept of a generalized complex space form is a natural generalization of a complex space form which has been discussed by Tricerri and Vanhecke in [9]. The authors stated the following theorem:

Theorem 1. An almost Hermitian manifold $M$ is a generalized complex space form if and only if $M$ is Einsteinian, weakly *-Einsteinian and Bochner-flat, i.e., $B=0$. Furthermore, a $2 n(n \geq 3)$-dimensional generalized complex space form is a real space form or a complex space form.

A $2 n(n \geq 2)$-dimensional almost Hermitian manifold $M=(M, J, g)$ is a generalized complex space form if the curvature tensor $R$ takes the following form:

$$
\begin{aligned}
R & =\frac{\tau+3 \tau^{*}}{16 n(n+1)}\left(\pi_{1}+\pi_{2}\right)+\frac{\tau-\tau^{*}}{16 n(n-1)}\left(3 \pi_{1}-\pi_{2}\right) \\
& =\frac{(2 n+1) \tau-3 \tau^{*}}{8 n(n-1)(n+1)} \pi_{1}+\frac{(2 n-1) \tau^{*}-\tau}{8 n(n-1)(n+1)} \pi_{2} .
\end{aligned}
$$

Observe that the results of Tricerri-Vanhecke's Theorem holds only for $2 n$ $\geq 6$. In [3], Lemence extended Theorem 1 to the 4-dimensional case. He had the following results:

Theorem 2. Let $M=(M, J, g)$ be a 4-dimensional generalized complex space form. Then $M$ is locally a real space form or globally conformal Kähler manifold. In the latter case, $\left(M, J, g^{*}\right)$ with $g^{*}=\left(3 \tau^{*}-\tau\right)^{\frac{2}{3}} g$ is a Kähler manifold, where $\tau$ and $\tau^{*}$ are the scalar curvature and the ${ }^{*}$-scalar curvature of $M$, respectively.

Theorem 3. Let $M=(M, J, g)$ be a compact 4-dimensional generalized complex space form. Then $M$ is a real space of constant non-positive sectional curvature or compact complex space form.

We now give our results on Bochner flat manifolds.

\section{Main Results}

Theorem 4. If $M=(M, J, g)$ be a 4-dimensional Bochner-flat, Einstein, weakly *Einstein manifold, then $M$ is a generalized complex space form.

Proof. When $n=2$, the expression for the Bochner tensor when $M$ is Einstein and weakly *-Einstein in (1) is reduced to

$$
B(X, Y, Z, W)=R(X, Y, Z, W)-\frac{5 \tau-3 \tau^{*}}{48} \pi_{1}(X, Y, Z, W)-\frac{3 \tau^{*}-\tau}{48} \pi_{2}(X, Y, Z, W) .
$$


Eileen May G. Cariaga, Richard S. Lemence, Marian P. Roque/ TJMCS Vol .2 No.4 (2011) 631-638

Since $M$ is Bochner-flat, then

$$
R(X, Y, Z, W)=\frac{5 \tau-3 \tau^{*}}{48} \pi_{1}(X, Y, Z, W)+\frac{3 \tau^{*}-\tau}{48} \pi_{2}(X, Y, Z, W)
$$

which satisfies (2) for $n=2$. Thus, $M$ is a generalized complex space form.

In [9], Tricerri and Vanhecke gave another expression for the Bochner tensor. They stated that if the curvature tensor $R$ of an almost Hermitian manifold satisfies $R(X, Y, Z, W)=R(X, Y, J Z, J W)$, then its corresponding Bochner tensor is given by

$$
B(R)=R-\frac{1}{2(n+2)}(\varphi+\psi)(\rho(R))+\frac{1}{4(n+1)(n+2)} \tau(R)\left(\pi_{1}+\pi_{2}\right) .
$$

This type of manifold is called an F-space. With this expression for $B$, we have the following characterization:

Theorem 5. Let $M=(M, J, g)$ be a connected Bochner-flat, Einsteinian F-space with real dimension $n \geq 3$. Then $M$ is a complex space form.

Proof. Observe that $\rho=\frac{\tau}{2 n} g$ since $M$ is Einsteinian. Therefore,

$$
\begin{aligned}
&(\varphi+\psi)(\rho(R))(X, Y, Z, W) \\
&=\frac{\tau}{2 n}(\varphi+\psi) g(X, Y, Z, W) \\
&=\frac{\tau}{2 n} \varphi(g)(X, Y, Z, W)+\frac{\tau}{2 n} \psi(g)(X, Y, Z, W) \\
&=\frac{\tau}{2 n}[g(X, Z) g(Y, W)+g(Y, W) g(X, Z)-g(X, W) g(Y, Z)-g(Y, Z) g(X, W) \\
&+2 g(X, J Y) g(Z, J W)+2 g(Z, J W) g(X, J Y)+g(X, J Z) g(Y, J W) \\
&+g(Y, J W) g(X, J Z)-g(X, J W) g(Y, J Z)-g(Y, J Z) g(X, J W)] \\
&= \frac{\tau}{n}[g(X, Z) g(Y, W)-g(Y, Z) g(X, W) \\
&+2 g(X, J Y) g(Z, J W)+g(X, J Z) g(Y, J W)-g(Y, J Z) g(X, J W)] \\
&= \frac{\tau}{n}[\{g(X, Z) g(Y, W)-g(Y, Z) g(X, W)\} \\
&+\{2 g(J X, Y) g(J Z, W)+g(J X, Z) g(J Y, W)-g(J Y, Z) g(J X, W)\}] \\
&= \frac{\tau}{n}\left[\pi_{1}(X, Y, Z, W)+\pi_{2}(X, Y, Z, W)\right] .
\end{aligned}
$$

Thus, the Bochner tensor will be reduced to 
Eileen May G. Cariaga, Richard S. Lemence, Marian P. Roque/ TJMCS Vol .2 No.4 (2011) 631-638

$$
\begin{aligned}
B & =R-\frac{1}{2(n+2)} \frac{\tau}{n}\left(\pi_{1}+\pi_{2}\right)+\frac{1}{4(n+1)(n+2)} \tau\left(\pi_{1}+\pi_{2}\right) \\
& =R-\frac{\tau}{4 n(n+1)}\left(\pi_{1}+\pi_{2}\right) .
\end{aligned}
$$

Since $M$ is Bochner-flat, then the curvature tensor $R$ takes the form

$$
\begin{aligned}
R(X, Y, Z, W) & =\frac{\tau}{4 n(n+1)}\left(\pi_{1}+\pi_{2}\right)(X, Y, Z, W) \\
& =\frac{\tau}{4 n(n+1)} \pi_{1}(X, Y, Z, W)+\frac{\tau}{4 n(n+1)} \pi_{2}(X, Y, Z, W) .
\end{aligned}
$$

In [9], it was stated that if $M$ is a connected almost Hermitian manifold with real dimension with real dimension $n \geq 3$ and Riemannian curvature tensor $R$ of the following form:

$$
R=f \pi_{1}+h \pi_{2}
$$

Where $f$ and $h$ are $C^{\infty}$ functions on $M$ such that $h$ is not identically zero, then $M$ is a complex space form. This still holds even if $f$ and $h$ are both constant functions. Hence, our assertion is proved.

Corollary 1. A connected Bochner-flat, Einstein F-space of real dimension $n \geq 3$ is a Kähler manifold with constant holomorphic sectional curvature $\frac{\tau}{4 n(n+1)}$, where $\tau$ is the scalar curvature.

Finally, we state an identity for the Riemannian curvature of a generalized complex space form.

Theorem 6. Let $M=(M, J, g)$ be a generalized complex space form. Then the curvature tensor $R$ satisfies the following curvature identity:

$$
R(X, Y, Z, W)=R(X, Y, J Z, J W)+R(X, J Y, Z, J W)+R(X, J Y, J Z, W)
$$

Proof. Let $\alpha=\frac{(2 n+1) \tau-3 \tau^{*}}{8 n\left(n^{2}-1\right)}$ and $\beta=\frac{(2 n-1) \tau^{*}-\tau}{8 n\left(n^{2}-1\right)}$. Then

$$
\begin{aligned}
R(X, Y, Z, W) & =\alpha \pi_{1}(X, Y, Z, W)+\beta \pi_{2}(X, Y, Z, W) \\
= & \alpha\{g(X, Z) \mathrm{g}(Y, W)-g(Y, Z) \mathrm{g}(X, W)\} \\
& +\beta\{2 g(J X, Y) \mathrm{g}(J Z, W)+g(J X, Z) \mathrm{g}(J Y, W)-g(J Y, Z) \mathrm{g}(J X, W)\} .
\end{aligned}
$$

The right-hand side of (4) can be expressed as

$$
\begin{aligned}
& R(X, Y, J Z, J W)+R(X, J Y, Z, J W)+R(X, J Y, J Z, W) \\
&= \alpha\{g(X, J Z) g(Y, J W)-g(Y, J Z) \mathrm{g}(X, J W)\} \\
&+\beta\{-2 g(J X, Y) \mathrm{g}(Z, J W)+g(J X, J Z) \mathrm{g}(J Y, J W)-g(J Y, J Z) \mathrm{g}(J X, J W)\}
\end{aligned}
$$


Eileen May G. Cariaga, Richard S. Lemence, Marian P. Roque/ TJMCS Vol .2 No.4 (2011) 631-638

$$
\begin{aligned}
& +\alpha\{g(X, Z) g(J Y, J W)-g(J Y, Z) g(X, J W)\} \\
& +\beta\{2 g(J X, J Y) g(J Z, J W)-g(J X, Z) g(Y, J W)+g(Y, Z) \mathrm{g}(J X, J W)\} \\
& +\alpha\{g(X, J Z) \mathrm{g}(J Y, W)-g(J Y, J Z) \mathrm{g}(X, W)\} \\
& +\beta\{-2 g(J X, J Y) \mathrm{g}(Z, W)-g(J X, J Z) \mathrm{g}(Y, W)+g(Y, J Z) \mathrm{g}(X X, W)\}
\end{aligned}
$$

We know that for an almost Hermitian manifold, $g(X X, J Y)=g(X, Y)$ and since $J^{2}=-1$, where 1 denotes the identity,

$$
g(J X, Y)=g\left(J^{2} X, J Y\right)=-g(X, J Y)
$$

Using these properties, we be able to identify the following and eliminate similar terms in (6).

$$
\begin{gathered}
g(X, J Z) g(Y, J W)=-g(X, J Z) g(J Y, W) \\
g(Y, J Z) g(X, J W)=-g(Y Y, Z) g(X, J W) \\
g(J X, J Z) g(J Y, J W)=g(J X, J Z) g(Y, W) \\
g(J Y, J Z) g(J X, J W)=-g(Y, Z) g(J X, J W) \\
2 g(J X, J Y) g(J Z, J W)=2 g(J X, J Y) g(Z, W) .
\end{gathered}
$$

Equation (6) now reduces to

$$
\begin{aligned}
& R(X, Y, J Z, J W)+R(X, J Y, Z, J W)+R(X, J Y, J Z, W) \\
& =\alpha\{g(X, Z) \mathrm{g}(J Y, J W)-g(J Y, J Z) \mathrm{g}(X, W)\} \\
& \quad+\beta\{-2 g(J X, Y) g(Z, J W)-g(J X, Z) \mathrm{g}(Y, J W)+g(J Y, J Z) \mathrm{g}(J X, W)\}
\end{aligned}
$$

Using the same mentioned properties about $g$ and $J$ in (6), finally we obtained

$$
\begin{aligned}
R(X, Y, J Z, J W)+R(X, J Y, Z, J W)+R(X, J Y, J Z, W) \\
=\alpha\{g(X, Z) \mathrm{g}(Y, W)-g(Y, Z) \mathrm{g}(X, W)\} \\
\quad+\beta\{2 g(J X, Y) \mathrm{g}(J Z, W)+g(J X, Z) \mathrm{g}(J Y, W)-g(J Y, Z) \mathrm{g}(J X, W)\}
\end{aligned}
$$

Finally, combining equations (5) and (8), we have the desired identity.

Acknowledgment. We would like to thank the referees for their comments and suggestions.

\section{References}

[1] T. Koda, Self-dual and anti-Self-dual Hermitian Surfaces, Kodai Math. $\mathrm{J}$. 10 (1987), 335-342.

[2] T. Koda and K. Sekigawa, Self-Dual Einstein Hermitian Surfaces, Advanced Studies in Pure Mathematics, Vol. 22 (1993) 123-131.

[3] R.S. Lemence, On Four-Dimensional Generalized Comples Space Forms, 
Eileen May G. Cariaga, Richard S. Lemence, Marian P. Roque/ TJMCS Vol .2 No.4 (2011) 631-638

Nihonkai Math. J., Vol. 15 (2004), 169-176.

[4] K. Matsuo, Pseudo-Bochner Curvature Tensor on Hermitian Manifolds, Colloquium Mathematicum, Vol. 80, No. 2 (1999), 201-209.

[5] K. Sekigawa, On some 4-dimensional compact almost Hermitian manifolds,

J. Ramanujan Math. Soc. 2 (1987), 101-116.

[6] K. Sekigawa and T. Koda, Compact Hermitian Surfaces of Pointwise Constant Holomorphic Sectional Curvature, Glasgow Math. J. 37 (1994), 343-349.

[7] S. Tachibana, On the Bochner Curvature Tensor, Natural Science Report, Ochanomizu University, Vol. 18. No. 1, 1967, 15- 19

[8] S. Tachibana and R.C. Liu, Notes on Kahlerian Metrics with Vanishing Bochner Curvature Tensor, Kodai Math. Sem. Rep. 22 (1970), 313-321.

[9] F. Tricerri and L. Vanhecke, Curvature Tensors on Almost Hermitian Manifolds, Transactions of the American Mathematical Society, Vol. 267,

No. 2, October 1981, 365-398.

[10] L. Vanhecke, The Bochner curvature tensor on almost Hermitian manifolds, Rend. Sem. Mat. Univ. Politec. Torino 34 (1975-76), 21-38.

[11] L. Vanhecke, On the Decomposition of curvature tensor fields on almost Hermitian manifolds, Proc. Conf. Differential Geometry, Michigan State Univ., East Lansing, Mich., 1976, pp. 16-33.

[12] L. Vanhecke and F. Bouten, Constant type for almost Hermitian manifolds, Bull. Math. Soc. Sci. Math. R.S. Roumanie 20 (1976), 415-422. 